\title{
Fuzzy Based PC-PUSH in CR-MANETs
}

\author{
S. Nejatian, ${ }^{1,2}$ S. K. Syed Yusof, ${ }^{1}$ N. M. Abdul Lattif, ${ }^{1}$ V. Rezaie, ${ }^{3}$ and V. Asadpour ${ }^{4}$ \\ ${ }^{1}$ UTM-MIMOS Center of Excellence, Faculty of Electrical Engineering, Universiti Teknologi Malaysia, 81310 Skudai, Johor, Malaysia \\ ${ }^{2}$ Department of Electrical Engineering, Faculty of Engineering, Islamic Azad University, Yasuj Branch, \\ Yasuj, Kohgiluyeh and Boyer-Ahmad Province 7591483587, Iran \\ ${ }^{3}$ Department of Mathematics, Faculty of Science, Islamic Azad University, Yasuj Branch, Yasuj, \\ Kohgiluyeh and Boyer-Ahmad Province 7591483587, Iran \\ ${ }^{4}$ Department of Electrical Engineering, University of North Dakota, Grand Forks, ND 58202, USA
}

Correspondence should be addressed to S. Nejatian; nsamad2@live.utm.my

Received 29 August 2014; Accepted 22 October 2014; Published 26 November 2014

Academic Editor: Ezzat G. Bakhoum

Copyright (C) 2014 S. Nejatian et al. This is an open access article distributed under the Creative Commons Attribution License, which permits unrestricted use, distribution, and reproduction in any medium, provided the original work is properly cited.

\begin{abstract}
In cognitive radio (CR), the secondary user (SU) needs to hand off its ongoing communication to an idle channel in order to avoid interference to the primary user (PU). Spectrum hand off issue becomes challenging in CR mobile ad hoc networks (CR-MANETs) because of the uncertainty in spectrum availability, broad range of spectrum bands and lack of central entity. The purpose of this study is to design a unified spectrum handoff (USH) scheme for CR-MANETs that considers the spectrum heterogeneity and its availability over time and space. A local flow hand off is performed when spectrum hand off cannot be carried out due to the SUs mobility. To improve further USH, preemptive unified spectrum handoff (PUSH) algorithm is proposed in which two different preemptive hand off threshold regions are defined. The PUSH algorithm also predicts the cognitive link availability considering the PU interference boundary. Although the PUSH scheme improves the hand off performance, the number of spectrum hand offs due to the PU activity should be reduced in this scheme. Therefore, the PC-PUSH (Power Controller-PUSH) scheme is proposed in which the fuzzy logic is used to improve the PUSH in terms of the number of spectrum handoffs because of the PU activity. The PC-PUSH decreases the interference to the PUs, while reducing the number of spectrum handoffs. The results show that the proposed scheme improves the link maintenance probability, decreases the hand off delay, and reduces the number of spectrum handoffs.
\end{abstract}

\section{Introduction}

Over the last few decades, the huge success of wireless applications has caused some spectrum bands being heavily used, especially unlicensed bands, such as ISM bands. This can be attributed to interference and poor network performance. On the other hand, recent studies by the Federal Communications Commission (FCC) have shown that a large amount of the licensed spectrum bands allocated through static assignment policies remains underutilized and inefficient. In fact, researchers have concluded that the traditional fixed spectrum allocation approach cannot continue to efficiently regulate the spectrum access. To address this situation, wireless devices operating in unlicensed bands can dynamically identify currently unused licensed spectrum bands for data communications and opportunistically access the unoccupied spectrum called spectrum holes or white spaces.

Cognitive capability with the enabling technology of cognitive radio (CR) allows wireless devices to use the spectrum holes. CR has the capability of completely changing its transmitter parameters (operating spectrum, modulation, and transmission power) based on interactions with the surrounding spectral environment. CR users, called secondary users (SUs) or unlicensed user, sense a wide spectrum range, identify the spectrum holes, and opportunistically access them when primary (or licensed) users (PUs) are idle [1]. PUs have been licensed to use the spectrum band and SUs must access the spectrum in a nonintrusive manner [2].

The latest trend in CR research area has seen an extensive research interest in mobile ad hoc networks (MANETs) to improve the spectrum efficiency by novel design techniques 
that simultaneously address the communication problems through various layers of the protocol stack. Enabling the CR technology in MANETs introduces the cognitive radio mobile ad hoc networks (CR-MANETs).

The uncertainty in the availability of spectrum holes is caused by the random appearance of PUs as well as the unpredictability of the SUs demand. The spectrum holes may shift over time and over space. In a CR system, the shifting of spectrum holes can be defined as spectrum mobility, which is cohesive to spectrum handoff. Spectrum handoff refers to the transfer of an ongoing data transmission of a CR user to another available spectrum band. In heterogeneous CR networks, a channel may be available over vast, mutually exclusive spectrum bands that present remarkable heterogeneity in terms of channel transmission range [3].

Spectrum handoff is extremely challenging in CR networks, especially in CR-MANETs, because of frequent topologic variations, limited power, limited channel transmission range, bandwidth constraints, and lack of the central controlling entity [4]. On the other hand, the available spectrum bands vary over time and space, while they are distributed nonadjacently over a broad frequency range. In a CR-MANET system, SU mobility and channel heterogeneity lead to performance degradation and frequent spectrum handoff during communication. Hence, the fluctuation of PU activity and the SU mobility in CR-MANETs have made the issue of maintaining optimal routes more complex.

Route failure has strong relationship with spectrum handoff. The route failure can be caused by spectrum mobility (PU activity), SU mobility, and channel heterogeneity. According to the failure type, different route recovery strategies need to be applied. Previous works on spectrum handoff in CRMANETs only consider the effect of PUs activity. Therefore, a unified spectrum handoff (USH) management for CRMANETs must be proposed that considers the effects of all of the factors mentioned above. In this work, a preemptive unified spectrum handoff (PUSH) method for CR-MANETs is proposed. PUSH considers the effects of mentioned factors on handoff initiation and management. In PUSH, two different preemptive handoff threshold regions were introduced and defined. A preemptive local flow handoff is performed when spectrum handoff cannot be carried out due to the SUs mobility. The PUSH algorithm also predicts the cognitive link availability and estimates the maximum link availability time considering the PU interference boundary. Although the PUSH scheme improves the handoff management scheme performance, the number of spectrum handoffs due to the $\mathrm{PU}$ activity should be reduced. Therefore, the PC-PUSH (Power Controller-PUSH) scheme is proposed in which the fuzzy logic is used to improve the performance of the PUSH in terms of the number of spectrum handoffs because of the PU activity.

\section{Related Works}

Song and Xie [5] proposed a proactive spectrum handoff configuration based on statistics of observed channel utilization. The network coordination and rendezvous issues are solved in this spectrum handoff scheme without using a common control channel. The collision among SUs is prevented through a distributed channel determination scheme. Giupponi and Pérez-Neira [6] proposed a fuzzy based spectrum handoff decision-making approach employing two fuzzy logic controllers. Each SU estimates the distances between itself and all the active PUs in the surrounding area using the first fuzzy logic controller. The other fuzzy logic controller determines whether the SU needs to perform a spectrum handoff and, in some cases, power control is used instead of handoff. Duan and Li proposed a spectrum handoff strategy in which the optimal spectrum band is chosen based on a multiplex criterion considering the estimated transmission time, the PU presence probability, and the spectrum availability time [7]. A cooperative spectrum sensing scheme is used to predict the spectrum idleness. A geolocation method is used to perform a spectrum handoff in the space domain. The simulation results indicated that the proposed spectrum handoff scheme outperformed conventional methods in terms of spectrum handoff delay in a per hop basis. However, channel heterogeneity parameters are not considered in the spectrum handoff. In [8], an established route from a source node $S$ to a destination node $D$ is considered. Different scenarios, which lead to the handoff initiation in this route, are also introduced. Considering these events, which are node mobility and spectrum mobility, the authors introduced a conceptual model for unified handoff management in CR-MANETs. Nejatian et al. [9] have characterized the availability of spectrum bands in CR-MANETs. The authors proved that the channel heterogeneity must be considered in terms of transmission range, because it increases the blocking probability of spectrum handoff. Based on their findings, a unified system, which considers the spectrum mobility in time and space domain as well as topology changing, must be investigated.

\section{Unified Spectrum Handoff (USH) Scheme}

In this section, first, the heterogeneous network architecture is described considering the specifics of the SUs, characteristics of the PUs, and the channel aspects of the network. Then, the USH scheme is introduced that considers the spectrum-aware handoff management based on the interactions between network layer and the physical layer. The proposed scheme is integrated with an algorithm to identify appropriate spectrum bands and calculate the channel availability time based on the channel qualities, the spectrum, and the node mobility.

3.1. System Description. It is assumed that the SU is equipped with multiradio, multichannel, and common control channel signaling features. The maximum number of channels accessible by the $\mathrm{SU}$ at a time is $C$. The $C$ channels are defined by the set of $\mathbf{T}$, which belong to the PU network. These channels are classified into different $L$ types according to their different transmission ranges. The set of each type is shown by $\mathbf{T}_{l}$ in which $\left|\mathbf{T}_{l}\right|=C_{l}$, which denotes the total number of channels for type $l$ and $C=C_{1}+C_{2}+\cdots+C_{L}$. Depending on the PU 
activity, any SU can access up to $C$ channels at any position in time. The number of detected channels of type $l$ by a node is $c_{l}$, and the total number of detected channels at a node is $c=c_{1}+c_{2}+\cdots+c_{L}$. The transmission range of channels of type $l$ is $R_{l}$.

3.2. Analytical Modeling of the USH Scheme. To propose the unified spectrum handoff management, different scenarios are introduced which cause spectrum handoff initiation through an established route in a CR-MANET. Suppose that a route from a source node $S$ to the destination node $D$ has been established. There are three different scenarios that initiate the spectrum handoff in this route.

Assume a pair of SU transmitter-receiver, which transmits and receives using communication channel. The activity of the PU in the neighbourhood of these nodes may cause the link between them to be failed. This route failure occurs once the PU starts its transmission or when one of the transmitterreceiver nodes enters the coverage area of the PU.

The mobility of the CR user can also lead to spectrum handoff due to spectrum heterogeneity and various channel transmission ranges. Again, assume a pair of SU transmitterreceiver, which transmits and receives using communication channel of type $l$, with transmission distance between them of less than $R_{l}$. When the SUs move and their distance exceeds $R_{l}$, the nodes must change and select another channel opportunistically. In this case, in order to support the communication link, the new chosen channel must have a transmission range longer than $R_{l}$.

In some cases, route failure occurs when either transmitter node or receiver node moves such that no channel can support their transmission. Before the route failure occurs, local flow handoff is performed. A local flow handoff can be from transmitter node to an intermediate node and finally joining receiver node. In this scenario, the unified local routing and spectrum handoff management system tries to solve the problem by finding a node as an intermediate node within the neighbouring area of the damaged links.

To analytically model the USH, the parameter $p$ is defined as the probability of single channel availability at a node by considering the PU activity with an alternating renewal twostate birth-death process with a death rate $\alpha$ and a birth rate $\beta$. The $P_{\text {car, } c}$ is defined as the probability of the existence of at least one idle channel for each hop in a route as follows [9]:

$$
\begin{aligned}
P_{\mathrm{car}, c}= & {\left[\sum_{i=1}^{L} \frac{\exp \left(-\lambda \pi R_{i-1}^{2} / 2\right)-\exp \left(-\lambda \pi R_{i}^{2} / 2\right)}{1-\exp (-N / 2)}\right.} \\
& \left.\times\left(1-\prod_{k=1}^{c-\sum_{j=0}^{i-1} c_{j}}\left(1-\left(\frac{\alpha_{k}}{\alpha_{k}+\beta_{k}}\right)^{2}\right)\right)\right]^{n-1} .
\end{aligned}
$$

Once the channel quality declines or PER (packet error rate) increases, the probability of successful packet transmission rate is decreased. Therefore, the SU decides to change the channel. Considering $P_{E}^{i}$ as the PER of channel of type $i$ and (1), the probability of successful packet routing in a route or between $n$ nodes is deduced to be

$$
p_{\mathrm{spr}, c}=\sum_{i=1}^{L} P_{\mathrm{car}, c}\left(1-P_{E}^{i}\right) .
$$

The probability of successful packet transmission in a hop or between two nodes is also found as

$$
p_{\mathrm{spt}, c}=\left.p_{\mathrm{spr}, c}\right|_{n=2}
$$

The parameter $P_{E}^{i}$ signifies the rate of dropped packets because of the variable channel conditions caused by factors such as fading and shadowing. The probability of unsuccessful packet transmission in a hop or between two nodes can be stated as

$$
p_{\mathrm{uspt}, c}=1-p_{\mathrm{spt}, c} \cdot
$$

To calculate the probability distribution of spectrum handoff and also model the spectrum handoff initiation in CRMANETs, the position case $D_{l-1 l}$ is defined as the case in which $R_{l-1}<d<R_{l}$, where $d$ is the length of the hop. Hereafter, for the sake of indexing convenience, the position case $D_{l-1 l}$ is referred to as $D_{l}$ throughout the thesis.

Figure 1 shows different Markov chains for spectrum handoff modelling based on the length of the hops. $\mathbf{P}$, which is a stochastic matrix and describes the Markov chain over the finite state space, is written as follows:

$$
\mathbf{P}=\left[\begin{array}{cccc}
P_{T_{11}} & \cdots & P_{T_{1 L-1}} & P_{T_{1 L}} \\
\vdots & \vdots & \vdots & \vdots \\
\cdots & P_{T_{i k}} & \cdots & P_{T_{i L}} \\
\vdots & \vdots & \vdots & \vdots \\
P_{T_{L 1}} & \cdots & P_{T_{L L-1}} & P_{T_{L L}}
\end{array}\right] .
$$

Here, the parameter $P_{T_{i k}}$ illustrates the probability of moving channel transition state from state $i$ to state $k$. The Markov chains have different numbers of states based on the different position cases $D_{l}$. When the length of the hop is less than $R_{1}$, or when the spectrum handoff occurs in the position case $D_{1}$, the two nodes involved in the current hop can select one of the available channels of any $L$ types. In the position case $D_{1}$, the Markov chain is as shown in Figure 1(a). In the case $D_{2}$, the nodes involved in spectrum handoff can select one channel among available channels from type $k$, in which $k \neq 1$, as shown in Figure 1(b). When the distance between the involved nodes in the spectrum handoff is according to position case $D_{L}$, the nodes can only select one channel among available channels of type $L$, as shown in Figure 1(c). The other situations can be determined based on the claims above.

Suppose that two nodes are communicating in a channel of type $k$. There are two different conditions where nodes continue their communication in the current spectrum pool. 


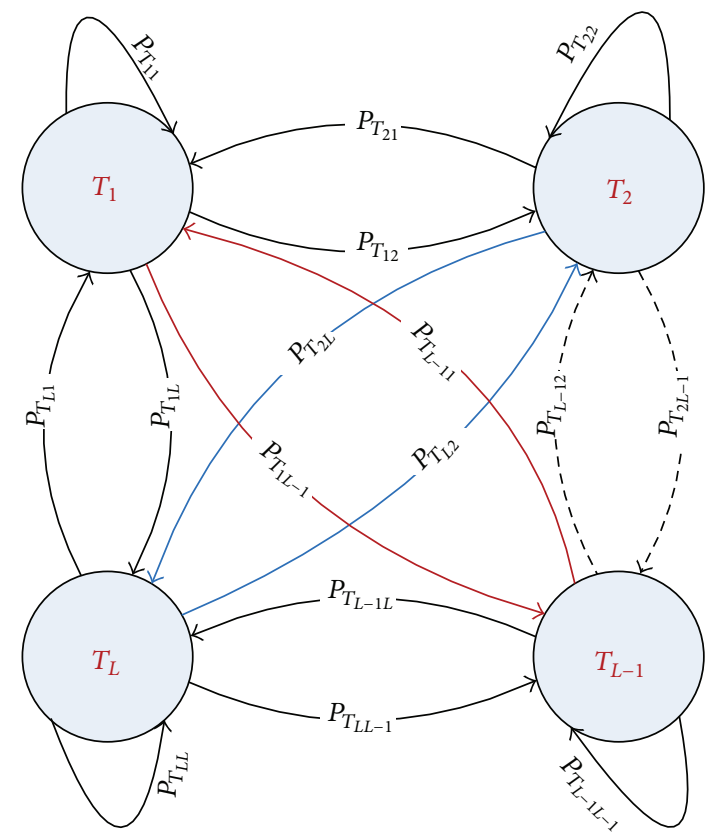

(a) Case $D_{1}$

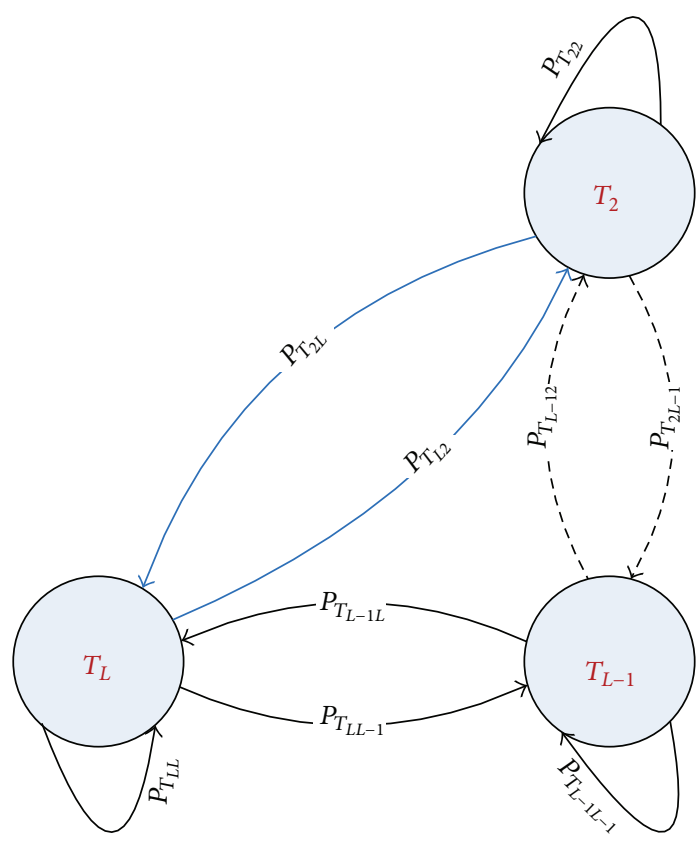

(b) Case $D_{2}$

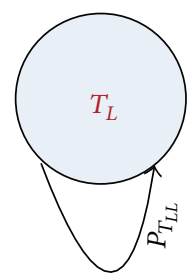

(c) Case $D_{L}$

FIGURE 1: Markov chains for spectrum handoff modeling based on the SUs distance.

These two conditions, in the position case of $D_{l}$, are as follows.

(i) The packet transmission is successful in the current channel of type $k$.

(ii) The packet transmission is not successful in the current channel of type $k$ but only successful on another channel of spectrum pool $k$.

Thus, the probability that the involved nodes in this hop do not switch their channel type, $P_{T_{k k}}$, is calculated as

$$
P_{T_{k k}, D_{l}}=p_{\mathrm{spt}, c_{k}}+\prod_{j=1, j \neq k}^{L} p_{\mathrm{uspt}, c_{j}} .
$$

There are also two conditions where two nodes, which are communicating on a channel of type $m$, switch their channel type to another channel of type $k$ such that $k \in$ $\{1,2, \ldots, L\} k \neq m$. These two conditions, in the position case $D_{l}$, are

(i) unsuccessful packet transmission in a channel of type $m$, but only successful transmission in channel of type $k$;

(ii) unsuccessful packet transmission in a channel of type $m$, but successful transmission in channel type sets:

$$
\mathbf{S}_{D_{l}} \subseteq \mathbf{T}, \quad\left|\mathbf{S}_{D_{l}}\right|=L_{D_{l}}<L .
$$

Based on the Markov chains, there are many possible channel type sets for $\mathbf{S}_{D_{l}}$. The channel of type $j$ can be chosen with an identical probability among the available channel types in the set of $\mathbf{S}_{D_{l}}$. Based on this explanation, the probability that the involved nodes in this hop switch their channel type, $P_{T_{m k}}$, is

$$
\begin{aligned}
& P_{T_{m k}, D_{l}}=\left[p_{\text {uspt }, c_{m}}\left(p_{\text {spt }, c_{k}}\left(\prod_{j=1, j \neq m, k}^{L} p_{\text {uspt }, c_{j}}\right)\right)\right] \\
& +\left[p _ { \text { uspt } , c _ { m } } \sum _ { \mathbf { S } _ { D _ { l } } \subseteq \mathbf { T } } \left(\left(\prod_{j=1,2, \ldots,\left.i\right|_{T_{j} \in s_{D_{l}} j \neq m}} p_{\text {sup }, c_{j}}\right.\right.\right. \\
& \left.\times \prod_{j=1,2, \ldots,\left.i\right|_{T_{j} \notin \mathrm{S}_{D} j \neq m}} p_{\mathrm{uspt}, c_{j}}\right) \\
& \left.\left.\times\left(\left|\mathbf{s}_{D_{l}}\right|\right)^{-1}\right)\right] .
\end{aligned}
$$

The row vector $\Pi_{D_{l}}^{\mathbf{P}}$, which is composed of $\Pi_{D_{l}}^{P}\left(T_{i}\right)$, demonstrates the steady state probability for $\mathbf{P}_{D_{l}}$ considering different hop lengths. The value of $\Pi_{D_{l}}^{P}\left(T_{i}\right)$ is calculated using the equations:

$$
\Pi_{D_{l}}^{\mathbf{P}} \times \mathbf{P}_{D_{l}}=\Pi_{D_{l}}^{\mathbf{P}}, \quad \sum_{i=1}^{L} \Pi_{D_{l}}^{P}\left(T_{i}\right)=1
$$


Ultimately, the steady state probabilities for various hop lengths for channel type $i$ are calculated as below:

$$
\Pi^{P}\left(T_{i}\right)=\sum_{l=1}^{L} \operatorname{Pr}\left(R_{l-1}<d<R_{l}\right) \Pi_{D_{l}}^{P}\left(T_{i}\right),
$$

where $\operatorname{Pr}\left(R_{l-1}<d<R_{l}\right)$ is calculated as [10]

$$
\begin{aligned}
\operatorname{Pr}\left(R_{l-1}<d<R_{l}\right) & =F_{d}\left(R_{l}\right)-F_{d}\left(R_{l-1}\right) \\
& =\frac{\exp \left(-\lambda \pi R_{l-1}^{2} / 2\right)-\exp \left(-\lambda \pi R_{l}^{2} / 2\right)}{1-\exp (-N / 2)} .
\end{aligned}
$$

The spectrum handoff procedure happens when the current channel can not support the data transmission. However, the spectrum handoff will not be initiated on any of the available channels due to other circumstances such as low channel transmission range as this can lead to transmission failure. Therefore, the probability of spectrum handoff can be formulated as

$$
P_{\mathrm{SH}}=\sum_{i=1}^{L} \Pi^{P}\left(T_{i}\right)\left[p_{\mathrm{uspt}, c_{i}}-\prod_{j=1}^{L} p_{\mathrm{uspt}, c_{j}}\right] .
$$

In (12), the term $\sum_{i=1}^{L} \Pi^{P}\left(T_{i}\right) \prod_{j=1}^{L} p_{\text {uspt, } c_{j}}$ is the probability that all available channels have unsuccessful packet transmission. In such a case, the troubled nodes do not perform spectrum handoff; they perform local flow handoff. Therefore, the probability of local flow handoff can be expressed as follows:

$$
P_{\mathrm{LH}}=\sum_{i=1}^{L} \Pi^{P}\left(T_{i}\right) \prod_{j=1}^{L} p_{\mathrm{uspt}, c_{j}} .
$$

The probability of successful spectrum handoff $\left(P_{\text {ssh }}\right)$ depends on the probability of successful packet transmission in a hop, $p_{\text {spt }, c}$. The link maintenance probability $\left(P_{\mathrm{LM}}\right)$ is defined as the probability that the link is successfully maintained in a hop or between troubled nodes, which is dependent on the probability of channel availability between two nodes. Thus, the link maintenance probability, considering only spectrum handoff, can be written as

$$
P_{\mathrm{LM}, \mathrm{SH}}=P_{\mathrm{SH}} \times p_{\mathrm{spt}, c} .
$$

When the link maintenance is not successful, despite performing spectrum handoff, local rerouting is performed. In this case, the probability of link maintenance can be represented as

$$
P_{\mathrm{LM}, \mathrm{LH}}=\left(\frac{N-1}{N}\right)\left(1-P_{\mathrm{LM}, \mathrm{SH}}\right) P_{\mathrm{LH}} \times p_{\mathrm{spt}, c} .
$$

Finally, the probability of link maintenance, considering the integration of local routing and spectrum handoff management, can be stated as

$$
P_{\mathrm{LM}, \mathrm{USH}}=P_{\mathrm{LM}, \mathrm{SH}}+P_{\mathrm{LM}, \mathrm{LH}} \cdot
$$

TABLE 1: Different parameters and their definitions used in the PUSH algorithm.

\begin{tabular}{ll}
\hline Symbol & Definition \\
\hline CSF & Channel switching flag \\
LAC & List of available and detected channels \\
HMF & Handoff metric flag \\
$T_{O}$ & Operation time \\
$t_{s}$ & Sensing time \\
NAC & Available channel number (channel ID) \\
RERR & Route error request \\
CSR & Channel switching request \\
CSA & Channel switching acknowledgment \\
HM & Handoff metric \\
\hline
\end{tabular}

\section{Preemptive Unified Spectrum Handoff (PUSH) Scheme}

In this section, the PUSH algorithm is proposed in which two different preemptive handoff threshold regions are defined and the cognitive link availability considering the PU interference boundary is predicted. The proposed handoff management scheme adapts to the unpredictable events, making a decision on the SUs data transmission without causing any interference with the PUs. The proposed algorithm for PUSH scheme is shown in the form of pseudocode presentation as shown in Pseudocode 1.

Table 1 defines the abbreviations and their definitions used in the pseudocode. The decision-making unit initiates the handoff based on the handoff threshold. When the spectrum handoff cannot be implemented in one hop, the LFH will be established to maintain communication.

In PUSH, PU activity is monitored by spectrum sensing and SU mobility is monitored by life time checking. If there is any problem with the SU mobility the HMF is activated and the algorithm will check the HM. Otherwise the algorithm checks for any available data to send. If there is no data to send, the algorithm remains in the event monitoring stage. When there is data to send, the PU activity is checked by the sensing part. The SUs continue their communication on the current channel until the expiration of the operation time $\left(T_{\mathrm{O}}\right)$. If there is any PU activity on the channel, the next channel for the handoff will be decided and the spectrum handoff will be performed. When the algorithm moves to check HM, if the HM is higher than the LHTH, only the spectrum handoff will be performed. The next channel for spectrum handoff must have a higher transmission range compared to the current channel. If the HM is lower than the LHTH, the troubled nodes try to find a suitable node as an intermediate node. Should there be no suitable node, the global flow handoff will be performed. Otherwise, the suitable node is chosen, the next channel will be decided, and the local flow handoff and consequently the spectrum handoff will be performed. 
Require: CSF $:=0, \mathrm{HMF}:=0, \mathrm{LAC}:=0 \varnothing, \mathrm{LFHREP}:=\varnothing, \mathrm{CSA}=\varnothing$

(1) check the HMF;

(2) if HMF $:=1$ then

(3) go to (42) //for checking the HM

(4) end if

(5) if there is no data to send then

(6) go back to (2) //for checking the HMF

(7) end if

(8) start scanning radio

(9) $\mathrm{PU}_{z_{*}} \mathrm{ON}_{-}=$sense (channel, $t_{s}$, switching)

(10) if $\mathrm{PU}_{z^{*}} \mathrm{ON}$ then

(11) defer for Channel_Switching_Delay

(12) for Next_Channel_Decision go to (24)

(13) $\mathrm{CSF}=1$

(14) go back to (5) //to check the availability of data for sending

(15) end if

(16) if (CSF) then

(17) Send notification to upper layers for spectrum handoff

(18) adapt channel parameters

(19) $\mathrm{CSF}:=0$

(20) end if

(21) start operation timer $\left(T_{o}\right)$

(22) transmit data till $\left(T_{o}\right)$ expires

(23) go back to (2)

(24) for $k:=1, k<C$ do

(25) if $\mathrm{PU}_{k} \neq \mathrm{ON}$ then

(26) $\mathrm{NAC}:=\mathrm{NAC}+1$

(27) $\mathrm{LAC}(\mathrm{NAC}):=k$

(28) end if

(29) end for

(30) if LAC $:=\emptyset$ then

(31) send RERR packet to the source node

(32) else $\{$ LAC $: \neq \emptyset\}$

(33) sending CSR //sending PU-HREQ

(34) go to (36)

(35) end if

(36) upon receiving CSA then

(37) if $\mathrm{CSA} \neq \emptyset$ then

(38) switch to the selected channel

(39) $\mathrm{HMF}:=0$

(40) go to (16)

(41) end if

(42) calculate the HM

(43) if $\mathrm{HM} \leq \mathrm{LHTH}$ then

(44) Start the LFHREQ timer, broadcast the LFHREQ and go to (49)

(45) else $\{\mathrm{HM} \leq \mathrm{SHTH}\}$

(46) go to (24) //for making a decision on the next channel

(47) end if

(48) upon the LFHREQ timer was expired then

(49) if LFHREP $:=\emptyset$ then

(50) broadcast the RERR to the source node

(51) else $\{$ LFHREP $\neq \emptyset\}$

(52) find the best candidate node with the $\max T_{\mathrm{PU}}$

(53) send the Local route HR to the local source through the best candidate intermediate node

(54) send the CSR to the candidate intermediate node

(55) go back to (36)

(56) end if

(57) end 


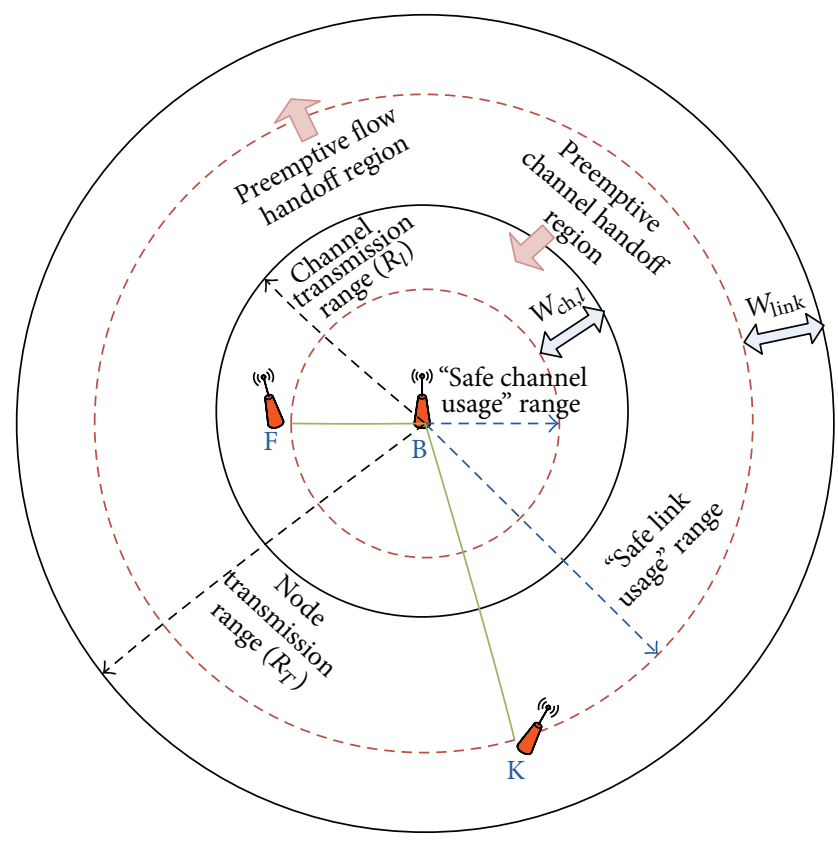

FIGURE 2: Different preemptive handoff regions.

4.1. Channel and Local Flow Handoff Prediction. A link is considered available if the two nodes associated with the link are within the transmission range of each other and out of interference region of any PU. In terms of transmission range, two different handoff regions are defined based on different handoff types. These two regions are the preemptive channel handoff region and the preemptive local flow handoff region. As illustrated in Figure 2, the first region is determined based on the channel transmission range, which is different for each channel type. The second region is determined by the node transmission range. Because the node transmission range depends on the wavelength of the transmission frequency, there is only one preemptive flow handoff region. Two different handoff thresholds are also defined related to each area. The first handoff threshold, which is related to the preemptive channel handoff region, is called the spectrum handoff threshold (SHTH).

The second handoff threshold is the LFH threshold (LHTH), which is related to the preemptive LFH region. These handoff thresholds are used to initiate the handoff due to the node mobility and channel quality degradation. As illustrated in Figure 2, nodes $B$ and $F$ are communicating with each other. When either node $B$ or node $F$ moves such that the current channel cannot support their communication, they must vacate the channel and transfer their transmission into another channel with a higher transmission range. In Figure 2, the $W_{\mathrm{ch}, l}$ is the warning distance for nodes communicating on a channel of type $l$. The $t_{w, l}$ which is the interval from the warning till the communication link break off needs to be greater than or equal to the necessary time for performing the handoff. Different handoff thresholds are related to the signal power threshold. Here, the signal power of hello packets is used to approximate the distance between the transmitter and receiver.
4.2. Channel Usage Time Prediction. Using the above proposed channel and local flow handoff prediction scheme with samples of the transmitted signal, the availability time is estimated without any movement information. Each SU keeps a neighbour signal information table (NSIT). The NSIT contains information about the condition of links between SU and its neighbours.

Based on [11], at least three packets are required to estimate the channel availability time. Suppose that at times $t_{1}, t_{2}$, and $t_{3}$ node $F$ receives the first, second, and third signal with respective power $P_{1}, P_{2}$, and $P_{3}$ from node $B$. The SU registers the signal power strength and reception time for each neighbour in the NSIT. When node $F$ receives packets from node $B$, it updates its NSIT array such that $P_{3}<P_{2}<P_{1}$ and $t_{1}<t_{2}<t_{3}$.

Assume at time $t$ the node $F$ will receive a signal with power equivalent to the $P_{s}$, and during time $t_{1}$ to $t$ the nodes $F$ and $B$ maintain their speeds and directions. Based on [11], the availability time is expressed as follows:

$$
t_{\mathrm{ava}}=\frac{\sqrt{b^{2}-4 a c}-b}{2 a},
$$

where

$$
\begin{gathered}
a=\Delta t_{2} \sqrt{P_{2} P_{s}} d, \quad b=\sqrt{P_{s}}\left(\left(\sqrt{P_{1}}-\sqrt{P_{2}}\right)-\Delta t_{2}^{2} \sqrt{P_{2}} d\right) \\
c=\Delta t_{2} \sqrt{P_{2} P_{s}}-\Delta t_{2} \sqrt{P_{1} P_{2}}, \quad \Delta t_{2}=t_{2}-t_{1}, \Delta t_{3}=t_{3}-t_{1} \\
d=\frac{\left(\sqrt{P_{1} P_{2}} \Delta t_{2}+\sqrt{P_{2} P_{3}} \Delta t_{3}-\sqrt{P_{1} P_{3}} \Delta t_{3}-\sqrt{P_{2} P_{3}} \Delta t_{2}\right)}{\left(\Delta t_{2} \Delta t_{3}^{2}-\Delta t_{3} \Delta t_{2}^{2}\right) \sqrt{P_{2} P_{3}}} .
\end{gathered}
$$

When the $P_{s}$ is replaced with the $P_{\mathrm{CTR}, l}$, the channel availability time between two communicating nodes for the channel of type $l\left(t_{\mathrm{ava}, l}\right)$ is an estimated value.

4.3. PU Interference Awareness and Link Availability. In terms of the PUs activity, a link is considered available when both two nodes associated with this link are out of the interference region of any PU in the network.

The PUSH scheme is aware of the PUs interference boundary and selects a suitable intermediate node with a high link availability time to handle the transmission in a LFH. To predict the link availability time considering the interference boundary of the PUs, the information about the distance between a mobile SU and each PU can be obtained through GPS or the path loss model. During the communication, the movement direction and velocity of mobile nodes can be considered constant in CR networks [12]. Based on [13], the $d_{\mathrm{PU}}$, which denotes the distance between the SU and the PU, can be expressed as

$$
d_{\mathrm{PU}}^{2}=\alpha t^{2}+\beta t+\gamma
$$

where $\alpha, \beta$, and $\gamma$ are constants and they are calculated using three measurement points, $\left(d_{\mathrm{PU}, 0}, t_{\mathrm{PU}, 0}\right),\left(d_{\mathrm{PU}, 1}, t_{\mathrm{PU}, 1}\right)$, and $\left(d_{\mathrm{PU}, 2}, t_{\mathrm{PU}, 2}\right)$. If $d_{\mathrm{PU}, \text { th }}$ shows the radius of the PUs interference boundary, then the maximum link accessibility period, $T_{\mathrm{PU}}$, 
with the PUs interference avoidance evaluated from $t_{\mathrm{PU}, 2}$ is expressed as

$$
T_{\mathrm{PU}}= \begin{cases}\frac{\sqrt{\beta^{2}+4 d_{\mathrm{PU}, \text { th }}^{2}-4 \alpha \gamma}-\beta}{2 a}-t_{\mathrm{PU}, 2}, & \Delta \geqslant 0, \Delta \geqslant \beta \\ \infty, & \text { otherwise, }\end{cases}
$$

where

$$
\Delta=\beta^{2}+4 \alpha d_{\mathrm{PU}, \mathrm{th}}^{2}-4 \alpha \gamma .
$$

Considering the link accessibility period with the PUs interference avoidance, the most suitable intermediate node is a node with maximum $T_{\mathrm{PU}}$.

4.4. Channel Allocation Scheme. To give a fair opportunity to the involved SUs in the longer hops, a weight matrix $\mathbf{W}$ with the size of $L \times L$ is proposed in the scheme. This weight matrix considers the hop length and the transmission range of the available channels, which can be expressed as follows:

$$
\mathbf{W}=\left[\begin{array}{cccc}
1 & \frac{1}{2} & \cdots & \frac{1}{L} \\
0 & 1 & \cdots & \frac{1}{L-1} \\
0 & 0 & \cdots & \frac{L}{L}
\end{array}\right] .
$$

The rows show the various position cases $D_{l}$ based on the hop length, and the column shows the available channel type l. Each element of $\mathbf{W}$ indicates a different location weight and is defined as follows:

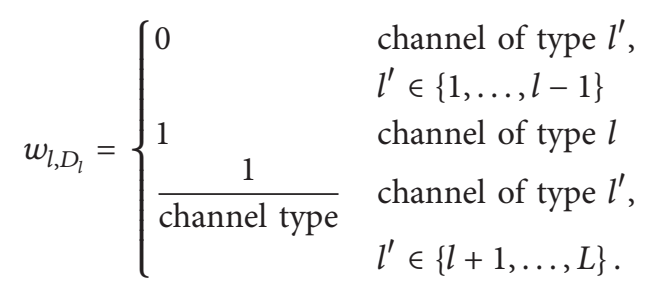

The hop length changing, which shows the SUs mobility, is considered in channel allocation scheme. The parameter $h_{l, D_{l}}$ is proposed to show the possibility of using the channel of type $l$, detected by both nodes in the current hop and the position case $D_{l}$ as follows:

$$
h_{l, D_{l}}= \begin{cases}1 & \text { channel of type } l^{\prime}, l^{\prime} \in\{l, \ldots, L\} \\ 0 & \text { channel of type } l^{\prime}, l^{\prime} \in\{1, \ldots, l-1\} .\end{cases}
$$

There are a number $C_{l}$ of channels of type $l$. Each channel of type $l$ is shown by $C_{l j}$. The channel $j$ of type $l$ can be used by the nodes belonging to the current hop, when it is available for both its member nodes. The parameter $\eta$ is proposed to show the possibility of using the channel $j$ of type $l$ for communicating in the current hop:

$$
\eta_{C_{l j}}= \begin{cases}1 & \begin{array}{l}
\text { channel } j \text { of type } l \text { is available for both } \\
\text { belonging to current hop } \\
0
\end{array} \\
\text { otherwise. }\end{cases}
$$

To avoid handing off the data transmission to a channel with a short availability time, the channel availability time must be greater than a threshold. Therefore, the parameter $\zeta$ is proposed to show the possibility of using the channel of type $l$ in the current hop:

$$
\zeta_{l, D_{l}}= \begin{cases}1 & \text { for } t_{\text {ava }, l, D_{l}} \geq t_{\text {th }} \\ 0 & \text { otherwise. }\end{cases}
$$

Finally, the channel allocation metric for channel $j$ of type $l$ can be derived as

$$
\operatorname{AM}_{C_{l j}}=\left(\zeta_{l, D_{l}}\right) \cdot\left(\eta_{C_{l j}}\right) \cdot\left(h_{l, D_{l}}\right) \cdot\left(w_{l, D_{l}}\right) \cdot\left(t_{\mathrm{ava}, l, D_{l}}\right) .
$$

Suppose that the set $\mathrm{CH}_{l, D_{l}}$ shows the set of channels of type $l$ detected by both nodes belonging to the current hop with position case $D_{l}$. The set $\mathrm{CH}_{D_{l}}$, which shows all the channels of different $L$ types detected by both nodes belonging to the current hop with position case $D_{l}$, can be expressed as

$$
\mathrm{CH}_{D_{l}}=\bigcup_{l^{\prime}=1}^{L} \mathrm{CH}_{l^{\prime}, D_{l}}
$$

The set of detected and useable channels for communication in the current hop with position case $D_{l}$ is defined as follows:

$$
\mathrm{CH}_{D_{l}, \text { usable }}=\bigcup_{l^{\prime}=1}^{L}\left[\bigcup_{C_{l^{\prime} j} \in \mathrm{CH}_{l^{\prime}, D_{l}}}\left(C_{l^{\prime} j}\right) \cdot\left(\eta_{C_{l^{\prime} j}}\right) \cdot\left(h_{l^{\prime}, D_{l}}\right)\right] .
$$

In the decision part, the channel $j$, which maximizes the channel allocation metric is formulated as below:

$$
\check{j}=\arg \max \left\{\mathrm{AM}_{C_{l j}}\right\}, \quad C_{l j} \subseteq \mathrm{CH}_{D_{l}, \text { usable }} .
$$

4.5. Handoff Initiation and Connectivity Management. To maintain end-to-end connectivity, topological variations and channel quality degradation due to node mobility are addressed using the handoff request (HREQ) packets. On the other hand, the PU handoff request (PU-HREQ) is used to address the variations in spectrum availability because of the PUs activity. The single-hop PU-HREQ packet informs the neighbour nodes that the PUs activity has been detected on a special channel. On the other hand, the HREQ is applied to inform the next hop node that the current link is breaking due to node mobility.

In terms of PU-HREQ, once a SU detects the PUs activity on a special channel, for example, channel $C_{l k}$, the SU discards all the entries through channel $C_{l k}$ and informs its neighbours that the channel is busy using a PU-HREQ. The PU-HREQ packet contains the available and detected channels of the current SU. The SUs that receive the PU-HREQ invalidate the entries through $C_{l k}$ that involve the PU-HREQ source. Using the NSIT based on the channel allocation scheme described before, the SU that receives the PU-HREQ finds the channel $j$ that maximizes the channel allocation 
metric described by (27). When the optimal channel is found, the node sends a handoff reply (PU-HREP) back. The PUHREP contains the new channel information to perform handoff and continue the data transmission. In terms of the topological variation due to node mobility or channel quality degradation, once the SU predicts the handoff, it broadcasts a single-hop channel HREQ (CH-HREQ) packet to its next hop node. When the next node receives the CH-HREQ packet, it makes a decision using the NSIT by a method similar to the procedure for PU-HREQ.

In LFH, when a SU as the local source (LS) predicts the presence of next hop node in the preemptive LFH region, it broadcasts a single-hop local flow HREQ (LFHREQ) containing the next hop nodes ID as the local destination (LD) through the CCC. LFH is performed to find an intermediate node (IN) for handling the data transmission responsibility. The selected node must be located in the transmission range of both nodes involved in current hop with the maximum link accessibility period $T_{\mathrm{PU}}$. A neighbouring node that receives the LFHREQ will search its NIT to determine whether the $\mathrm{LD}$ is in its NIT. If node LD has been registered as a neighbor node in its NIT, the IN estimates the $T_{\mathrm{PU}}$ with all the active PUs in its surrounding area. It finds the minimum $T_{\mathrm{PU}}$ among all the PUs, and it sends the LFH reply (LFHREP) packet back to the LS. The LFHREP contains the minimum $T_{\mathrm{PU}}$ of the current node. The LS compares all the $T_{\mathrm{PU}}$ information received from its neighbors. Then, it selects the best node (BN) among candidate nodes through which nodes LS and LD can maintain the longest life time avoiding the PUs interference. Then, LS sends a handoff request (HR) to the local destination through the BN using CCC. The HR contains information such as the ID of the LS, the ID of the $\mathrm{LD}$, and the channel availability list of the current node. Once the $\mathrm{BN}$ receives the $\mathrm{HR}$, then we have the following.

(i) The $\mathrm{BN}$ compares its own available channels $C_{\mathrm{ava}, \mathrm{BN}}$ with the available channels of the local source $C_{\text {ava,LS }}$ in HREQ. The usable channel set in this hop can be expressed as

$$
\mathrm{CH}_{D_{l}, \text { usable }}=\bigcup_{l^{\prime}=1}^{L}\left[\left(C_{\mathrm{ava}, \mathrm{BN}, l^{\prime}} \cap C_{\mathrm{ava}, \mathrm{LS}, l^{\prime}}\right) \cdot h_{l^{\prime}, D_{l}}\right] \text {. }
$$

(ii) The $\mathrm{BN}$ determines the channel $j$ which maximizes the channel allocation metric from the channel set $\mathrm{CH}_{D_{l} \text {, usable }}$ using (27).

(iii) Let $j$ be the selected channel, node BN updates HREQ with its own information, the ID of node LS, the ID of node LD, and its available channel list.

(iv) Send the HREQ to the local destination node LD through the common control channel.

When the LD node receives the $\mathrm{HR}$, similar to the $\mathrm{BN}$, it selects a proper channel for its upper hop. The local destination sends the handoff acknowledgment (HA) packet back to the LS. The HA message sets up a new route, and the routing tables in all three nodes are updated. Once the new route is established, the data flow will be passed along the new route. In the case that the LFH is not possible, the global flow handoff is performed by the source node.

Although the PUSH scheme improves the handoff management scheme performance, the number of spectrum handoffs due to the PU activity should be reduced more. In the next section, the PC-PUSH scheme is proposed in which the fuzzy logic is used to improve the performance of the $\mathrm{PUSH}$ in terms of the number of spectrum handoffs because of the PU activity.

\section{Fuzzy Based PC-PUSH Scheme}

Here, the PC-PUSH algorithm is proposed. In PC-PUSH, each SU is equipped with an FB-PC strategy that is an adaptive decision-making unit. The FB-PC strategy improves the spectrum handoff performance of the SU by adapting to the network conditions, traffic changes, and consequently controlling the interference to the PUs. The proposed PCPUSH scheme adapts to unpredictable events and makes a decision on the SUs data transmission without causing any interference to the PUs. Therefore, CR must change its parameters according to the decision before transmitting the signal. The changes in pseudocode presentation of PC-PUSH scheme compared to the PUSH scheme are as shown in Pseudocode 2.

As it can be seen in line 10 of the proposed pseudocode for the PC-PUSH scheme, when the PU activity is detected, the algorithm will be directed to the decision-making part (line 11). The decision policy, which is a fuzzy decision strategy, qualitatively determines whether SU should switch its ongoing data transmission to another unused channel or it can change its power level in order to avoid interference with PU and stay in the current channel. While in the PUSH scheme, once the occupied band is claimed by PU, the SU switchs its ongoing data transmission to another unused channel. In the next subsection, the FB-PC strategy will be explained.

5.1. Fuzzy Based (FB) Power Controller (PC) Strategy. The proposed FB-PC strategy tries to keep the transmission power of the SU at an acceptable level in order to avoid interference with PU while ensuring proper signal quality at the receiving end. When the SU decides to stay in the current spectrum band, its transmission power is balanced between a minimum required transmission power $\left(P_{\text {min,req }}\right)$ and a maximum acceptable transmission power $\left(P_{\text {max,acp }}\right)$ considering the allowed interference level at the PU. The minimum required transmission power $\left(P_{\text {min,req }}\right)$ determines the SU transmit power just enough to satisfy the required SINR at SU receiver.

Same as the general fuzzy logic controller [14], the proposed FB-PC strategy consists of four modules named as fuzzifier, fuzzy rule base, inference engine, and defuzzifier. The controlling operation is started by measuring the variables that show the relevant conditions of the controlled process. There are two input linguistic variables in FB-PC strategy.

As Figure 3 shows, the $P_{\text {max,acp }}$ as the maximum acceptable transmission power at the SU location and the $\mathrm{SINR}_{\text {diff }}$ 


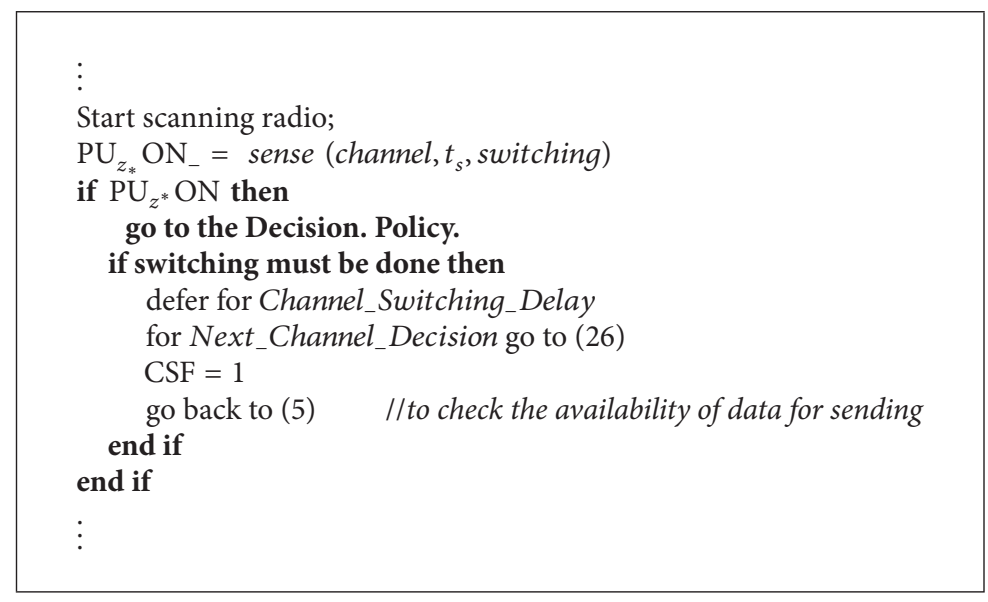

Pseudocode 2

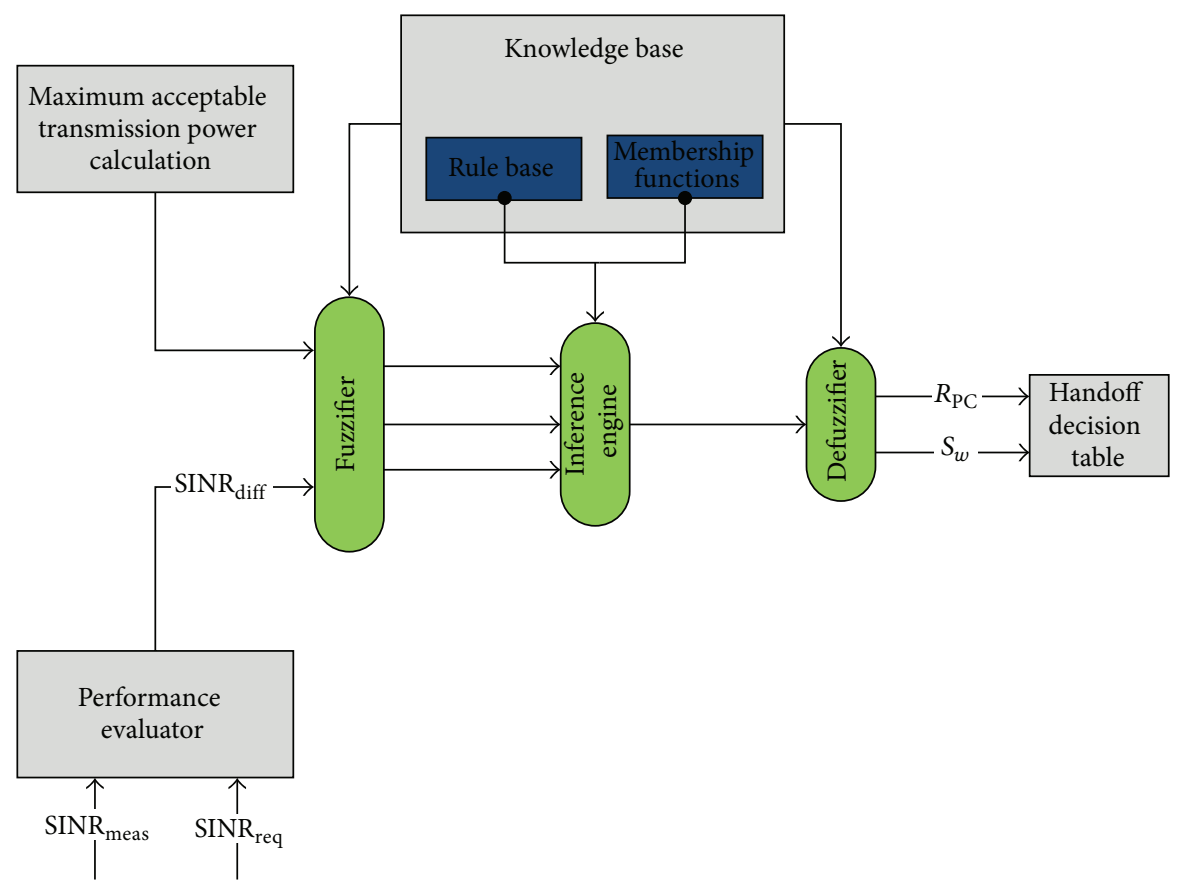

Figure 3: Fuzzy based Power Controller strategy.

as the difference between required $\operatorname{SINR}\left(\operatorname{SINR}_{\text {req }}\right)$ and the measured SINR $\left(\mathrm{SINR}_{\text {meas }}\right)$ at the SU receiver are two input linguistic variables. In the next step, these measurements are transferred into fuzzy sets (fuzzification) to show the uncertainties in measurements. Then, using these measurements, the interference engine evaluates the control rules stored in the fuzzy rule base module. Finally, the results of this evaluation which can be several fuzzy sets determined on the universe of discourse of possible actions are converted to crisp values (defuzzification). Each input linguistic variable is characterized by a term set of three fuzzy sets, "Low," "Medium," and "High."

The FB-PC strategy is characterized by two outputs. The first output (Sw) shows whether the channel switching must be performed or not. The second output $\left(R_{\mathrm{PC}}\right)$, which shows the transmit power control ratio, indicates how the SU transmission power should be modified when the channel switching is not necessary. The term set of $S w$ output linguistic variable consists of three fuzzy sets, "Yes," "Probably Yes," and "No." While the term set of $R_{\mathrm{PC}}$ output linguistic variable consists of three fuzzy sets, "Low," "Medium," and "High." The fuzzy inference rules can be easily derived from these term sets.

To calculate the $P_{\text {max acp }}$, the path loss model is used. Consider $I_{\mathrm{PU} \text {,lim }}$ as a predefined interference temperature limit for an active PU, which implies that both the SU and PU can use the licensed spectrum band simultaneously, but the SU must guarantee an interference temperature level perceived by the PU less than $I_{\mathrm{PU}, \mathrm{lim}}$ [15]. It means that if the total interference level originating from noise floor (NF), 
other PUs activities, and so on is kept below the $I_{\mathrm{PU}, \mathrm{lim}}$, the SU can use the spectrum bands opportunistically. Therefore, an allowed interference temperature for SU $\left(I_{\mathrm{SU}, \max }\right)$ to the target $\mathrm{PU}$ is as follows:

$$
I_{\mathrm{SU}, \max }=I_{\mathrm{PU}, \mathrm{lim}}-I-\mathrm{NF} \text {. }
$$

in which $I$ shows the interference from other PUs and NF indicates noise floor power level.

Using the path loss model, the $I_{\mathrm{SU} \text {, max }}$ is converted to the corresponding $P_{\text {max,acp }}$ at a SU's location as follows [16]:

$$
I_{\mathrm{SU}, \max }=P_{\max , \mathrm{acp}}\left[\frac{d_{0}}{d_{\mathrm{PU}}}\right]^{n},
$$

where $n$ is a number typically between 2 and $4, d_{0}$ shows the reference distance, and $d_{\mathrm{PU}}$ denotes the distance between the SU and the target PU explained in Section 4.3. Therefore, considering (33), it can be concluded that

$$
P_{\text {max }, \text { acp }}=I_{\mathrm{SU}, \max }\left[\frac{d_{0}}{d_{\mathrm{PU}}}\right]^{-n} .
$$

Finally, considering (33) and (34), the maximum acceptable transmission power is calculated as follows:

$$
P_{\text {max acp }}[\mathrm{dB}]=I_{\mathrm{SU}, \max }[\mathrm{dB}]-10 \cdot n \cdot \log _{10}\left[\frac{d_{0}}{d_{\mathrm{PU}}}\right][\mathrm{dB}] \text {. }
$$

\section{Results and Discussions}

In this section, the performance of the proposed unified handoff management is analyzed and evaluated through extensive simulations considering the various conditions of the network, traffics, and mobility parameters. The throughput of the whole system will be analyzed to check for any significant improvement by introducing unified spectrum handoff management scheme. Here, a patch of NS-2 for cognitive radio cognitive network (CRCN), which supports various functionalities of the cognitive radio networks, is used with some modifications for the extensive simulation of the proposed scheme. The AODV [17] routing protocol is used for route formation over CR-MANETs.

The performance improvement achieved by the proposed method is demonstrated using four different versions of the handoff management schemes that are defined as the spectrum handoff (SH), the USH, the PUSH, and the PC-PUSH algorithm. The terminology applied for the simulation study is described as follows. The first scheme, SH, only deploys the spectrum handoff, while the remaining three schemes deploy the unified spectrum handoff in which the local flow handoff will be added to the system. One of these three schemes, USH, does not consider the preemptive handoff threshold, whereas two others, PUSH and PC-PUSH, consider the preemptive handoff threshold for the preemptive handoff region and also consider the PU interference boundary. Finally, the PC-PUSH performs the FB-PC strategy to avoid spectrum handoff.
The transmission range of the static PUs on their occupied channels is set to $200 \mathrm{~m}$, and the PUs activity is modeled as a two-stage on/off procedure with an exponential distribution. An average of 40 trial runs is used in which the locations of the PU nodes are randomly chosen. Depending on the simulation scenario, a different number of mobile SUs are distributed in the network. For SUs mobility, random waypoint model is applied with a speed uniformly distributed from minimal speed $1 \mathrm{~m} / \mathrm{s}$ to maximal speed $10 \mathrm{~m} / \mathrm{s}$ in which the pause time is set to 0.0 seconds. In some simulation scenarios, the SUs speed is fixed to a predetermined point. The sensing time and operation time are fixed to $0.1 \mathrm{sec}$ and $0.9 \mathrm{sec}$, respectively. The total number of available channels $C=10$ is classified into 2 different types. The center carrier frequencies of available data channels are $200 \mathrm{MHz}$ and $800 \mathrm{MHz}$. The transmission ranges of different channel types and the node transmission range are set to $R_{1}=75 \mathrm{~m}, R_{2}=125 \mathrm{~m}$, and $R_{T}=150 \mathrm{~m}$, respectively. The mobile SUs are distributed in a network within a $2000 \mathrm{~m} \times 2000 \mathrm{~m}$ area. Both the $W_{\mathrm{ch}, l}$, which is the warning distance for the preemptive channel handoff region for nodes communicating on a channel of type $l$, and $W_{\text {link }}$, which is defined as the threshold distance for the preemptive local flow handoff region, are set to $12 \mathrm{~m}$. Both channel usage time and the link life time threshold are set to 12 seconds.

6.1. Number of Spectrum Handoffs, Link Maintenance, and Spectrum Handoff Delay Performance. In this subsection, the SUs route maintenance probability, number of required spectrum handoffs, and spectrum handoff delay are investigated.

The probability of unsuccessful link maintenance is defined as the probability of spectrum handoff blocking $\left(P_{\mathrm{hb}}\right)$. Figure 4 evaluates the different handoff management schemes in terms of the $\left(P_{\mathrm{hb}}\right)$. Based on the analytical model, Figure 4(a) compares the probability of unsuccessful rerout$\operatorname{ing}\left(P_{\mathrm{usrr}}\right)$, the $\left(P_{\mathrm{hb}, \mathrm{SH}}\right)$, and the $\left(P_{\mathrm{hb}, \mathrm{USH}}\right)$, considering various numbers of SU nodes in the network with a number of hops equal to 5 . Based on this figure, the USH scheme outperforms the $\mathrm{SH}$ scheme in terms of link maintenance probability. The probability of link maintenance in the USH scheme is also significantly higher than the probability of successful rerouting. As the number of SUs in the network increases, the probability of handoff blocking decreases because the probability of finding the proper nodes, which work as the relay to perform local rerouting, increases.

Figure 4(b) shows the handoff blocking probability based on both analytical model and real time simulation considering various arrival rates of the PUs in the network. Based on this figure, in the analytical model, the unified handoff management scheme outperforms the scheme deploying only spectrum handoff in terms of link maintenance probability, which is also verified by the simulation results. The simulation results indicate that the spectrum handoff blocking probability in the PUSH scheme is lower than the probability of the spectrum handoff blocking in the scheme deploying only spectrum handoff. This is caused by LFH deployment in PUSH. Moreover, the $P_{\mathrm{hb}, \mathrm{PUSH}}$ is lower than the probability of handoff blocking in the scheme deploying USH. 


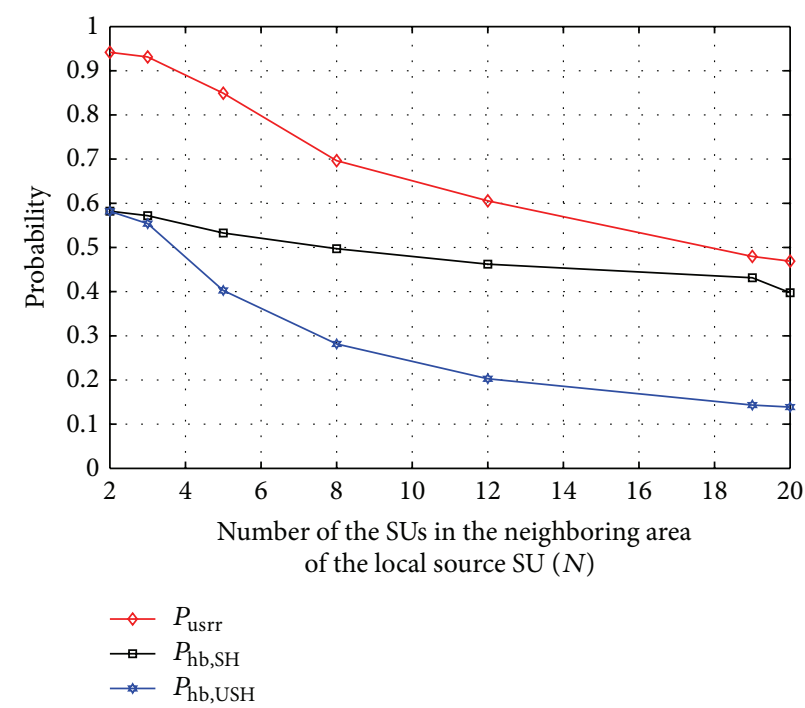

(a) Versus number of the SUs

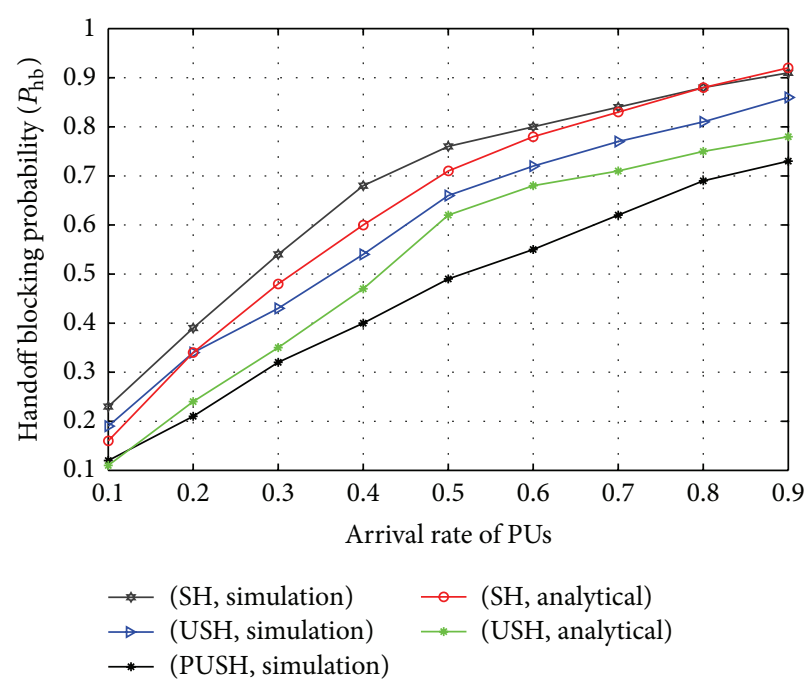

(b) Versus the arrival rate of the PUs

FIGURE 4: Comparison of different handoff management schemes performance in terms of $\left(P_{\mathrm{hb}}\right)$.

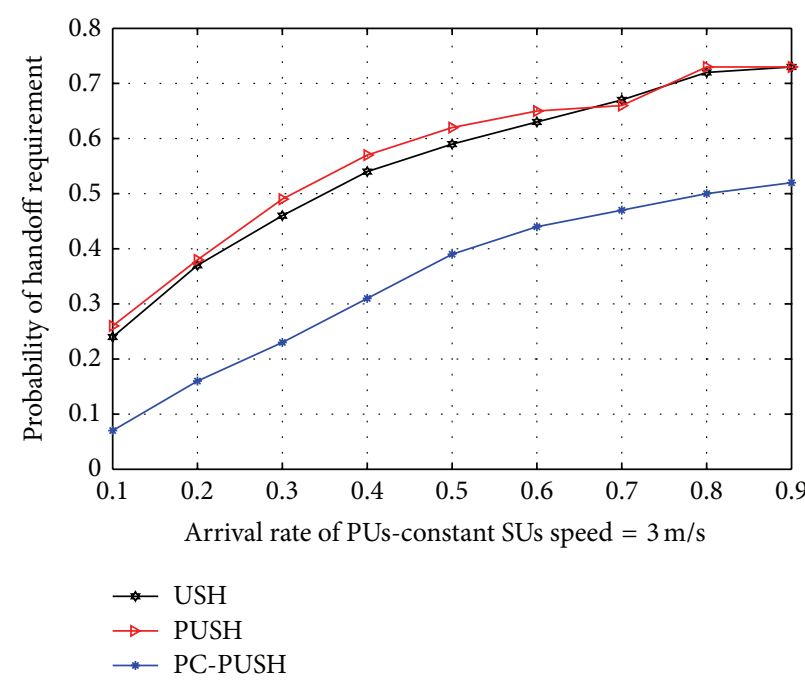

(a) Versus the arrival rate of PUs

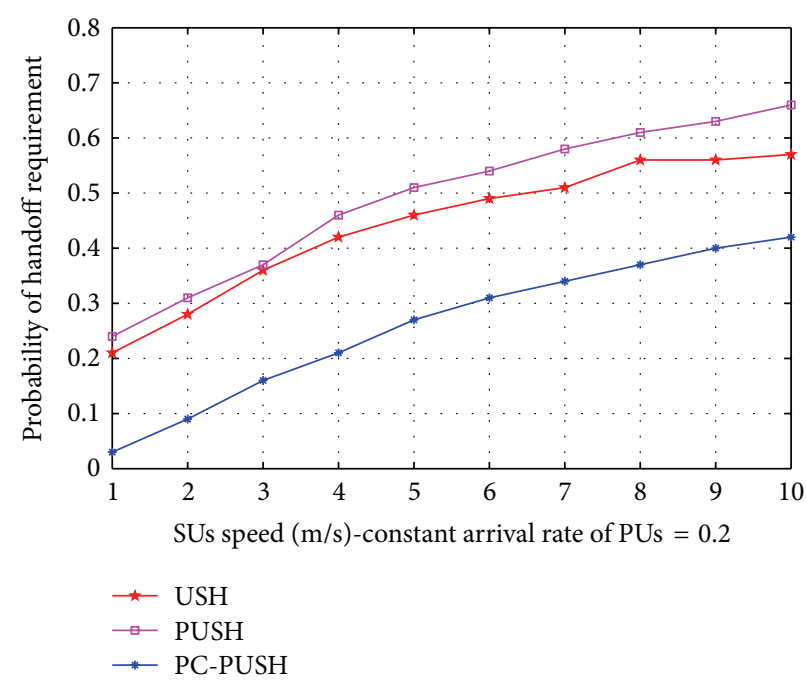

(b) Versus the velocity of SUs

FIGURE 5: The expected amount of handoff requirements for the SUs.

Figure 5 shows the expected amount of handoff requirement for the SUs. To evaluate the effects of the PU's activity and the SU's mobility, the PU's arrival rate and SU's velocity vary in Figures 5(a) and 5(b), respectively. From this figure, it can be stated that the FB-PC strategy reduces the number of required handoffs in the handoff management scheme significantly. Based on Figure 5(b), rather than the PU activity, the SU's mobility has an important effect on the performance of the CR network. Hence, the mobility of SUs must be considered in the handoff management schemes.

To investigate the effects of the preemptive handoff management and FB PC strategy on the amount of handoff delay, Figure 6 shows the simulation results of the average spectrum handoff delay under different network conditions.
The PU's arrival rate and SU's velocity vary in Figures 6(a) and 6(b), respectively. From these figures, it can be stated that, by using the preemptive unified handoff management scheme, the average spectrum handoff delay is reduced significantly. It can also be stated that the FB-PC strategy reduces the amount of handoff delay because the number of spectrum handoffs is decreased and the total handoff delay will be reduced. Here, the preemptive strategy reduces the number of spectrum handoffs arising from the SU mobility, while the FB-PC strategy reduces the number of spectrum handoffs originating from the PU activity.

Figure 7 shows the route maintenance probability for the SUs versus the arrival rate of PUs. Based on this figure, it is stated that the proposed unified handoff approach efficiently 


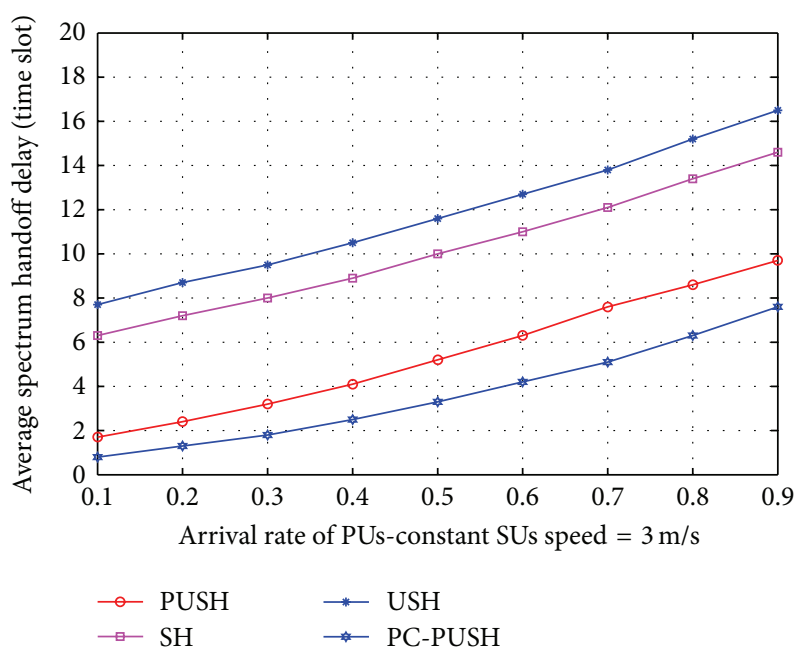

(a) Versus the arrival rate of PUs

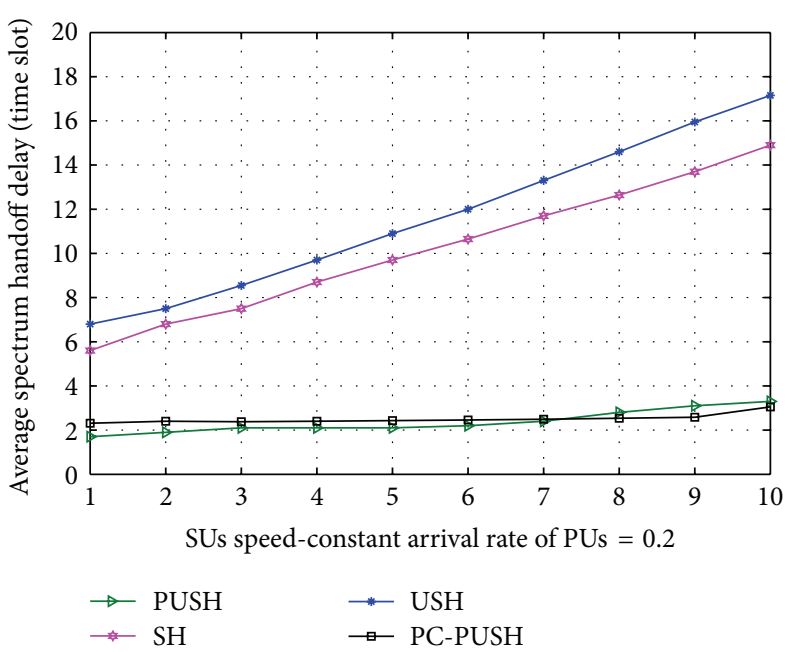

(b) Versus the velocity of SUs

FIGURE 6: Average spectrum handoff delay under different network conditions.

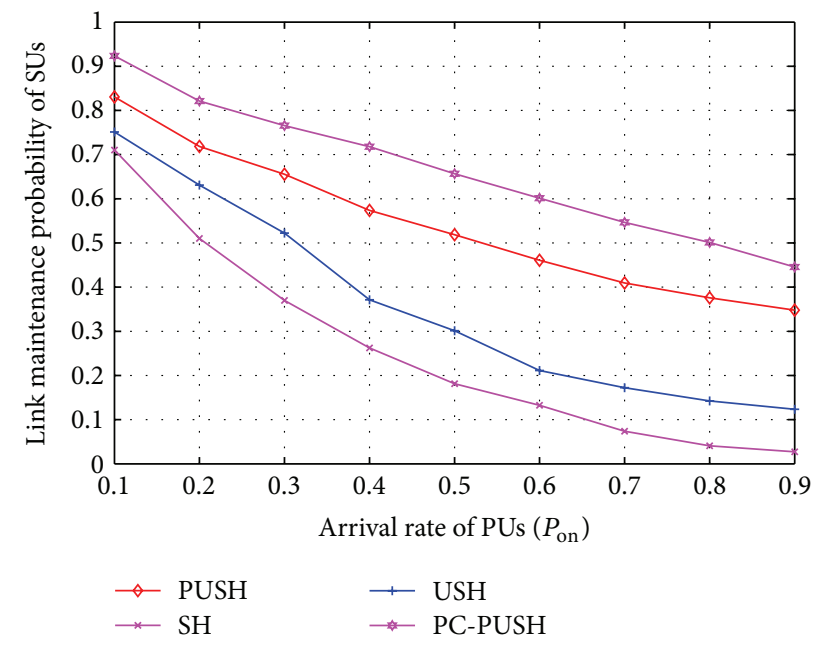

FIGURE 7: Route maintenance probability for the SUs versus the arrival rate of PUs.

improves the route maintenance probability. This figure also shows that the proposed PC-PUSH scheme outperforms other handoff management schemes in terms of maintaining the established routes.

6.2. SU Throughput. In this subsection, the performance of different handoff management schemes in terms of SU's throughput is analyzed and evaluated. Figure 8 shows the SU throughput considering different network conditions. Figure 8(a) shows the $\mathrm{SU}$ throughput under various numbers of PU channels. As the number of PU channels increases, the SUs throughput increases because more channels can be exploited by the SUs for data transmissions. In this figure, the FB PC-PUSH scheme outperforms the three other schemes in terms of SU throughput. Figure 8(b) shows the $\mathrm{SU}$ throughput versus the arrival rate of PUs. This figure confirms that the PC-PUSH improves the SU throughput performance for increasing PU arrival rate in comparison with both PUSH and USH schemes. This is because of the FB PC strategy conducted by the PC-PUSH scheme that decreases the number of handoffs increases the probability of successful link maintenance and also decreases the handoff delay. Furthermore, there is an obvious difference in SUs throughput for the $\mathrm{SH}$ and the $\mathrm{PUSH}$ protocols as the former does not account for the local flow handoff and also PU activity regions avoidance.

\section{Conclusion}

Spectrum handoff management is an open issue in CR networks. It is particularly challenging in CR-MANETs. In CR-MANETs, the available spectrum bands vary over time and space, while they are distributed nonadjacently over a broad frequency range. However, in CR-MANETs, the fluctuation of PU activity and the SU mobility make the issue of maintaining optimal routes more complex. In this work, a unified spectrum handoff management scheme is presented where it considers spectrum mobility in the time and space domains and considers the network topology variations in CR-MANETs. A network architecture that considers the heterogeneous spectrum availability and its variation over time, space, and distributed nodes is proposed in which the handoff is performed preemptively. The proposed PUSH algorithm predicts the cognitive link availability considering the PU interference boundary. Although the PUSH scheme improves the handoff management scheme performance, the number of spectrum handoffs due to the PU activity should be reduced more. Therefore, the PC-PUSH (Power Controller-PUSH) scheme is proposed in which the fuzzy logic is used to improve the performance of the PUSH in terms of the number of spectrum handoffs because of the PU activity. The PC-PUSH decreases the interference with the PUs, while reducing the number of spectrum handoffs. The 


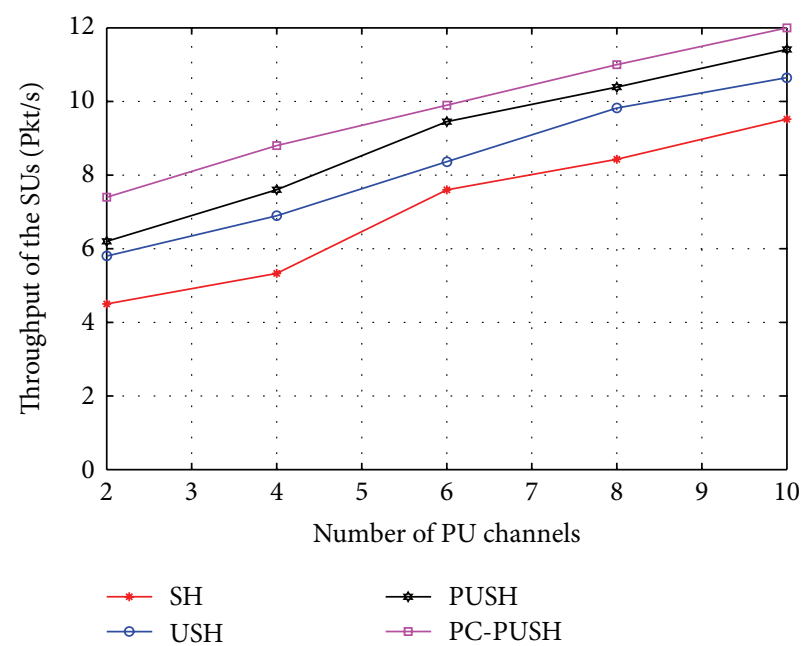

(a) Versus the numbers of PU channels

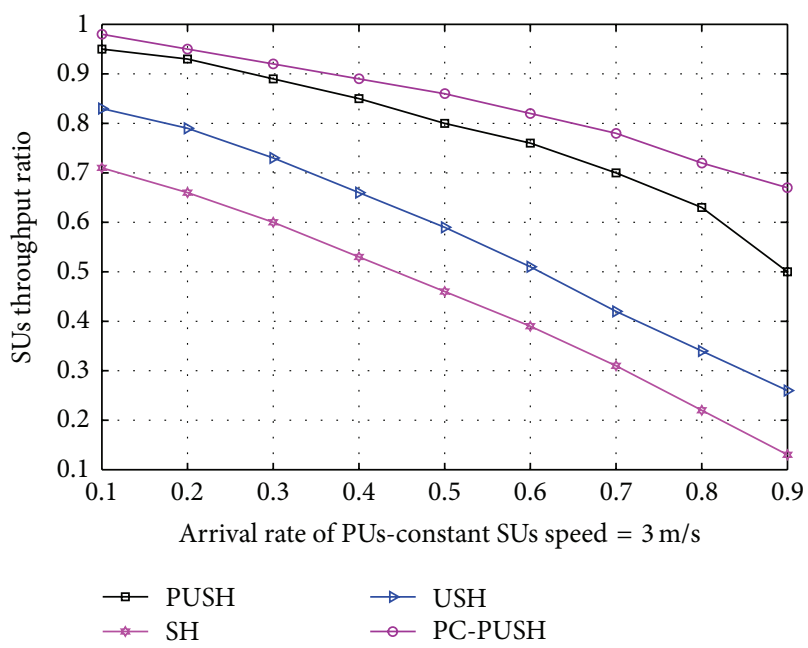

(b) Versus the arrival rate of PUs

FIGURE 8: Comparison of the performance of different handoff management schemes in terms of the SUs throughput versus different network conditions.

results of both analytical model and simulation study verify that the proposed scheme improves the link maintenance probability, decreases the handoff delay, and reduces the number of spectrum handoffs.

\section{Conflict of Interests}

The authors declare that there is no conflict of interests regarding the publication of this paper.

\section{Acknowledgments}

The authors would like to thank all those who contributed toward making this research successful. Also, they would like to thank all the reviewers for their insightful comments. The authors wish to express their gratitude to the Ministry of Higher Education (MOHE) and Research Management Center (RMC) of Universiti Teknologi Malaysia (UTM) for the financial support of this project under GUP Research Grant no. Q.J130000.2509.07H04.

\section{References}

[1] Y. Ding and L. Xiao, "Channel allocation in multi-channel wireless mesh networks," Computer Communications, vol. 34, no. 7, pp. 803-815, 2011.

[2] D. Lu, X. Huang, W. Zhang, and J. Fan, "Interference-aware spectrum handover for cognitive radio networks," Wireless Communications and Mobile Computing, vol. 14, no. 11, pp. 1099-1112, 2014.

[3] G. Ning, J. Duan, J. Su, and D. Qiu, "Spectrum sharing based on spectrum heterogeneity and multi-hop handoff in centralized cognitive radio networks," in Proceedings of the 20th Annual Wireless and Optical Communications Conference (WOCC '11), pp. 1-6, April 2011.

[4] S. Nejatian, S. K. Syed-Yusof, N. M. A. Latiff, N. Fisal, and V. Asadpour, "Handoff management in cognitive radio networks: concepts, protocols, metrics and challenges," International Review on Computers and Software, vol. 7, no. 6, pp. 2993-3006, 2012.

[5] Y. Song and J. Xie, "ProSpect: A proactive spectrum handoff framework for cognitive radio Ad hoc networks without common control channel," IEEE Transactions on Mobile Computing, vol. 11, no. 7, pp. 1127-1139, 2012.

[6] L. Giupponi and A. I. Pérez-Neira, "Fuzzy-based spectrum handoff in cognitive radio networks," in Proceedings of the $3 r d$ International Conference on Cognitive Radio Oriented Wireless Networks and Communications (CrownCom '08), pp. 1-6, Singapore, May 2008.

[7] J. Duan and Y. Li, "An optimal spectrum handoff scheme for cognitive radio mobile Ad hoc networks," Advances in Electrical and Computer Engineering, vol. 11, no. 3, pp. 11-16, 2011.

[8] S. Nejatian, S. K. Syed-Yusof, N. M. Abdul Latiff, and V. Asadpour, "Proactive integrated handoff management in CRMANETs: a conceptual model," in Proceedings of the IEEE Symposium on Wireless Technology and Applications (ISWTA '12), pp. 32-37, Bandung, Indonesia, September 2012.

[9] S. Nejatian, S. K. Syed-Yusof, N. Abdul Latiff, and V. Asadpour, "Characterization of spectrum mobility and channel availability in crmanets," in Proceedings of the 1st International Conference on Advances in Mobile Network and Communication (MNC '12), 2012.

[10] T.-C. Hou and V. O. K. Li, "Transmission range control in multihop packet radio networks," IEEE Transactions on Communications, vol. 34, no. 1, pp. 38-44, 1986.

[11] L. Qin and T. Kunz, "Increasing packet delivery ratio in dsr by link prediction," in Proceedings of the 36th Annual Hawaii International Conference, pp. 23-31, 2003.

[12] Q. Guan, F. R. Yu, and S. Jiang, "Prediction-based topology control and routing in cognitive radio mobile ad hoc networks," in Proceedings of the IEEE Conference on Computer Communications Workshops (INFOCOM '10), pp. 1-6, March 2010.

[13] S. Jiang, D. He, and J. Rao, "A prediction-based link availability estimation for routing metrics in manets," IEEE/ACM Transactions on Networking, vol. 13, no. 6, pp. 1302-1312, 2005. 
[14] J. M. Mendel, "Fuzzy logic systems for engineering: a tutorial," Proceedings of the IEEE, vol. 83, no. 3, pp. 345-377, 1995.

[15] I. F. Akyildiz, W.-Y. Lee, M. C. Vuran, and S. Mohanty, "Next generation/dynamic spectrum access/cognitive radio wireless networks: a survey," Computer Networks, vol. 50, no. 13, pp. 2127-2159, 2006.

[16] T. Goff, N. Abu-Ghazaleh, D. Phatak, and R. Kahvecioglu, "Preemptive routing in ad hoc networks," Journal of Parallel and Distributed Computing, vol. 63, no. 2, pp. 123-140, 2003.

[17] C. E. Perkins and E. M. Royer, "Ad-hoc on-demand distance vector routing," in Proceedings of the 2nd IEEE Workshop on Mobile Computing Systems and Applications (WMCSA '99), pp. 90-100, New Orleans, La, USA, February 1999. 


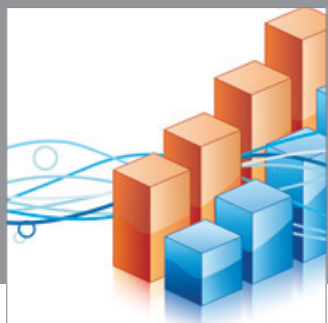

Advances in

Operations Research

mansans

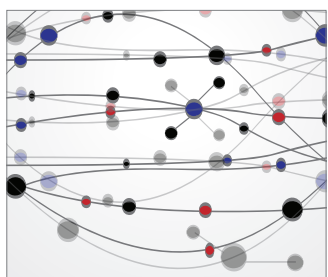

The Scientific World Journal
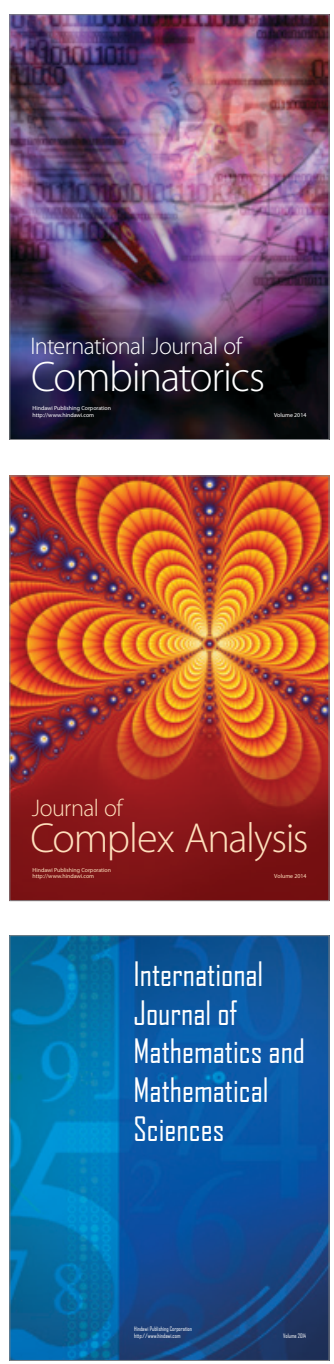
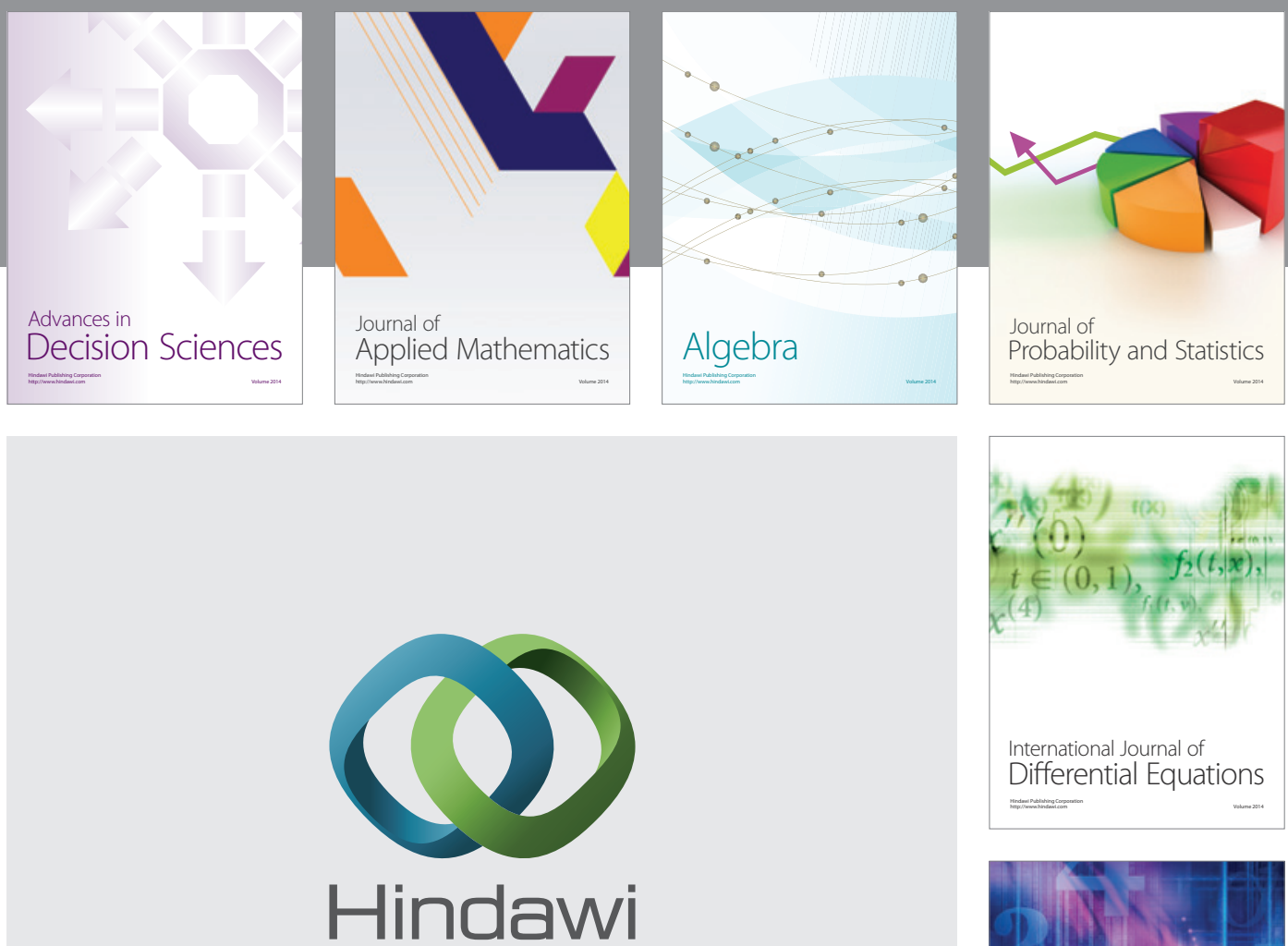

Submit your manuscripts at http://www.hindawi.com
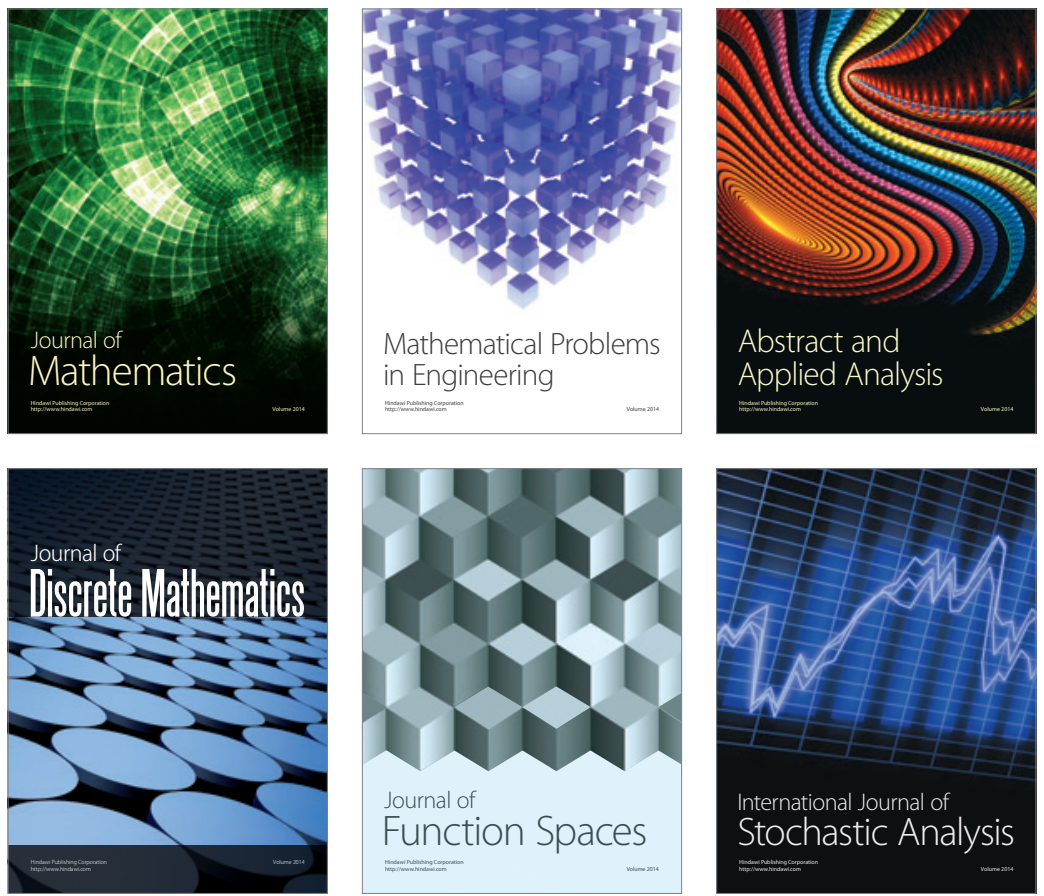

Journal of

Function Spaces

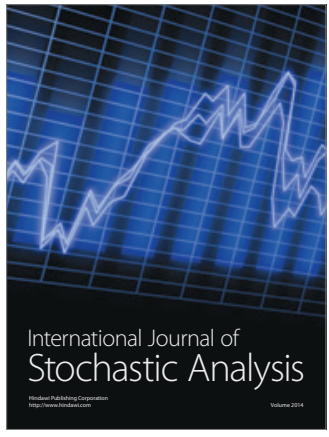

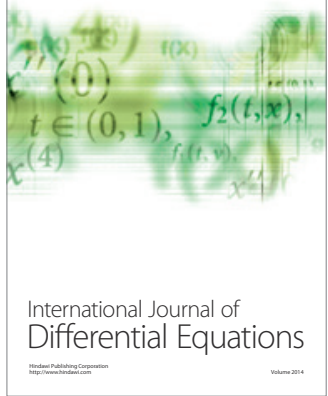
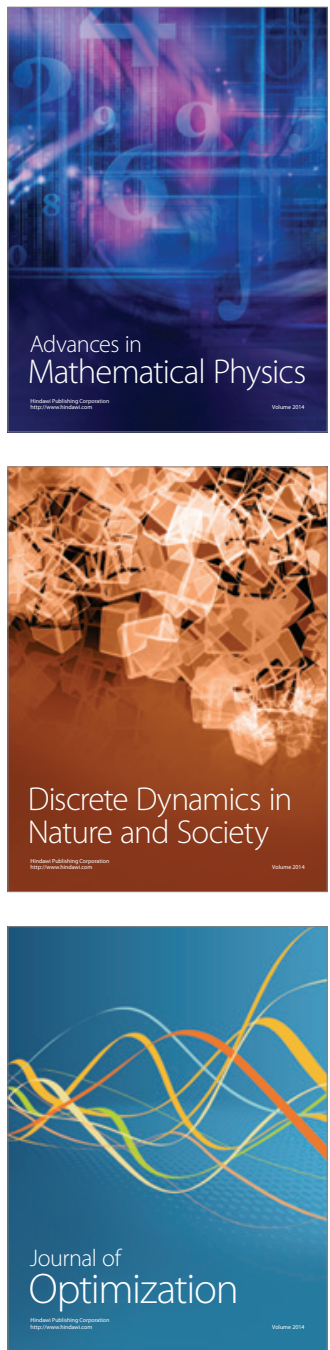